\title{
Differential effects of atorvastatin on autophagy in ischemic and nonischemic myocardium in Ossabaw swine with metabolic syndrome
}

\author{
Ashraf A. Sabe, MD, Nassrene Y. Elmadhun, MD, Ahmed A. Sadek, BS, Louis M. Chu, MD, \\ Cesario Bianchi, MD, PhD, and Frank W. Sellke, MD
}

\begin{abstract}
Objectives: The perioperative administration of pleomorphic statin drugs has been implicated in improving outcomes after cardiac surgery. Adaptive autophagy is a highly conserved cellular process that allows for the elimination of dysfunctional cell components in response to stress and survival under starving conditions. We sought to investigate the effects of the statin drug atorvastatin on autophagy in ischemic and nonischemic myocardia using a clinically relevant porcine model of metabolic syndrome.
\end{abstract}

\begin{abstract}
Methods: Male Ossabaw swine were fed a regular diet $(\mathrm{n}=8)$, a high-cholesterol $\operatorname{diet}(\mathrm{n}=8)$, or a high-cholesterol diet with supplemental atorvastatin $(1.5 \mathrm{mg} / \mathrm{kg} / \mathrm{d})(\mathrm{n}=8)$. After 14 weeks, all animals underwent surgical placement of an ameroid constrictor to the circumflex coronary artery to induce chronic ischemia. Nonischemic and ischemic myocardia were harvested 6 months after initiation of the diet and processed for Western blotting.
\end{abstract}

Results: In the nonischemic myocardium, Western blot results demonstrate that a high cholesterol diet resulted in a statistically significant decrease in autophagy as indicated by an increase in mammalian target of rapamycin and the accumulation of several essential autophagy markers, including Beclin-1, light chain 3B-I, and light chain 3B-II. Atorvastatin supplementation prevented these changes and resulted in an increase in autophagy as indicated by a decrease in autophagy flux marker P62. In the ischemic myocardium, atorvastatin had the opposite effect, with a decrease in autophagy flux as indicated by an increase in p62 and an accumulation of light chain 3B-I, light chain B-II, and lysosome-associated membrane protein 2.

Conclusions: Atorvastatin administration has differential effects on autophagy in ischemic and nonischemic myocardia. In the setting of metabolic syndrome, atorvastatin stimulates autophagy in nonischemic myocardium while partly inhibiting autophagy in ischemic myocardium. The differential regulation on autophagy may, in part, explain the cardioprotective effect of statins in both ischemic and nonischemic myocardia, and these findings may have implications in the setting of cardiac surgery. (J Thorac Cardiovasc Surg 2014;148:3172-8)

See related commentary on pages $3178-9$.

Autophagy is an adaptive and essential cellular process that eliminates damaged or harmful cellular components. ${ }^{1}$ When optimally regulated, autophagy has been shown to be cardioprotective. ${ }^{2}$ In patients undergoing cardiac surgery, clinical studies have demonstrated that surgical

From the Division of Cardiothoracic Surgery, Cardiovascular Research Center, Warren Alpert School of Medicine, Brown University, Providence, RI.

Funding for this research was provided by the National Heart, Lung, and Blood Institute (R01HL46716 and R01HL69024 to Dr Sellke) and National Institutes of Health Training Grant 5T32-HL094300-03 (to Drs Sabe, Elmadhun, and $\mathrm{Chu}$ ). Additional funding for this animal model was from National Institutes of Health Grant RR013223.

Disclosures: Frank W. Sellke reports consulting fees from CSL Behring and the Medicines Company. All other authors have nothing to disclose with regard to commercial support.

Received for publication April 23, 2014; revisions received July 26, 2014; accepted for publication July 29, 2014; available ahead of print Sept 17, 2014.

Address for reprints: Frank W. Sellke, MD, Division of Cardiothoracic Surgery, Cardiovascular Research Center, Warren Alpert Medical School of Brown University, 2 Dudley St, MOC 360, Providence, RI 02905 (E-mail: fsellke@ lifespan.org).

0022-5223/\$36.00

Copyright (c) 2014 by The American Association for Thoracic Surgery

http://dx.doi.org/10.1016/j.jtcvs.2014.07.104 risk scores and crossclamp times are correlated to alterations in autophagy flux during surgery. ${ }^{3}$ Impaired autophagy has been shown to result in unfavorable cardiac remodeling and increased rates of postoperative atrial fibrillation in patients undergoing cardiac surgery. ${ }^{4}$ In cardiac myocytes, autophagy is upregulated as a result of cellular stress from hypoxia and chronic ischemia and is significantly downregulated in the setting of caloric excess and metabolic syndrome (Figure 1). Several physiologic pathways affect autophagy signaling and induction. When autophagy is induced, a double-membrane " $\mathrm{C}$ "-shaped structure is formed and closes, resulting in an autophagosome. The autophagosome fuses with a lysosome forming an autophagolysosome, which ultimately envelops and removes unfavorable cellular components, such as damaged organelles and accumulated amino acids ${ }^{2,5}$ (Figure 1). Although autophagy in excess may lead to cell death, when appropriately regulated, autophagy allows for cellular salvage and repair, in contrast to apoptosis and necrosis. ${ }^{4,6,7}$ Thus, exploring therapies that may favorably affect autophagy are of special interest in the heart, where cardiomyocytes have a limited capacity for regeneration. A number of novel and commonly used medications have 


\section{Abbreviations and Acronyms \\ IM = ischemic myocardium \\ LAMP-2 $=$ lysosome-associated membrane protein 2 \\ LC3B-I = light chain 3B-I \\ LC3B-II = light chain 3B-II \\ mTOR = mammalian target of rapamycin \\ NIM = nonischemic myocardium \\ $\mathrm{OC} \quad=$ regular diet control group \\ $\mathrm{OHC}=$ high cholesterol control group \\ OHCS = high cholesterol control group \\ supplemented with daily oral atorvastatin}

been reported to regulate autophagy in the heart, including resveratrol, rapamycin, and statins. ${ }^{2,8,9}$

Statins are of particular interest given their vast clinical use and significant cardioprotective effects. ${ }^{10-12}$ They have been clearly shown to reduce morbidity and mortality in patients with coronary artery disease, and there is increasing evidence that perioperative statin use is associated with a significant reduction in major adverse outcomes after cardiac surgery. ${ }^{11-16}$ In recent years, statin use has been demonstrated to regulate autophagy activity in tumor cells, vascular endothelial cells, and cardiac cells. ${ }^{17-19}$ Statins also have been shown to affect angiogenesis by promoting endothelial cell growth and migration, although the mechanism remains under investigation. ${ }^{20}$ In a large animal model, we previously demonstrated that atorvastatin modulates myocardial angiogenesis, despite increasing oxidative stress in the setting of chronic ischemia. ${ }^{21}$ These findings suggest a compensatory intracellular response preventing oxidative damage to endothelial cells, such as autophagy.

It is important to note that many of the aforementioned studies have been performed in small, relatively healthy animal models. Although these studies are important, they are often difficult to translate in a clinically relevant manner. Given the increase in obesity and diabetes, we have been using a large animal (porcine) model of metabolic syndrome to better mimic the complex patients seen in the population with cardiac disease. ${ }^{22,23}$ Although no animal model perfectly represents human pathophysiology, pigs have similar hemodynamics, drug pharmacokinetics, and cardiac anatomy. Also, pig hearts have similar coronary circulation and, like humans, lack preexisting collateral vessels and spontaneously develop atherosclerosis. ${ }^{24}$ Thus, to further investigate the effects of atorvastatin on autophagy, we used a clinically relevant, porcine model of metabolic syndrome and chronic myocardial ischemia. We hypothesized that atorvastatin would prevent the inhibition of autophagy resulting from a high-fat diet leading to metabolic syndrome.

\section{MATERIALS AND METHODS Animal Model and Surgical Interventions}

Ossabaw swine (Purdue Ossabaw Facility, Indiana University, Indianapolis, Ind) were divided into 3 groups based on a daily diet fed over a 14-week period. The regular diet control group (OC, $\mathrm{n}=8$ ) was fed a diet consisting of "regular" chow. The high cholesterol control group $(\mathrm{OHC}, \mathrm{n}=8$ ) was given daily feedings of $500 \mathrm{~g}$ of a high cholesterol diet as previously described. ${ }^{21}$ Animals in the experimental group were fed the same diet as the OHC animals but were supplemented with daily oral atorvastatin $(1.5 \mathrm{mg} / \mathrm{kg} / \mathrm{d}$; OHCS, $\mathrm{n}=8)$ (Pfizer Inc, New York, NY). After 14 weeks, animals underwent surgical placement of a titanium ameroid constrictor (Research Instruments SW, Escondito, Calif) on the proximal left circumflex coronary artery. Six months after ameroid placement, the animals were euthanized and their hearts were harvested. Myocardial tissue collected from chronically ischemic myocardium (IM) and nonischemic myocardium (NIM) was rapidly frozen in liquid nitrogen. Tissues used for analysis in this study are from animals that were reported in our previous study. ${ }^{21}$ The methods for weight measurements, dextrose challenge, anesthesia, and surgical interventions have been described. ${ }^{21}$ All experiments were approved by the Institutional Animal Care and Use Committee of the Rhode Island Hospital. Animals were cared for in compliance with the Principles of Laboratory Animal Care formulated by the National Society for Medical Research and the Guide for the Care and Use of Laboratory Animals.

\section{Protein Expression}

Protein lysates were prepared from NIM and IM. Lysate preparation, Western blotting, image capture, and band quantification methods were performed as previously described. Lysates transferred to polyvinylidene difluoride membranes (Millipore, Bedford, Mass) were incubated overnight at $4{ }^{\circ} \mathrm{C}$ in primary antibodies at dilutions recommended by the manufacturer. Primary antibodies used were mammalian target of rapamycin (mTOR), Beclin-1, light chain 3B-I (LC3B-I), light chain 3B-II (LC3B-II) (all from Cell Signaling Technology, Danvers, Mass), and lysosome-associated membrane protein 2 (LAMP-2) (Life Technologies, Grand Island, NY). Membranes were incubated with the appropriate horseradish peroxidase-linked secondary antibody at room temperature for approximately 1 hour (Jackson ImmunoResearch Laboratories Inc, West Grove, Pa). All membranes were probed with glyceraldehyde 3-phosphate dehydrogenase (Cell Signaling Technology) to correct for protein loading error.

\section{Data Analysis}

All Western blot results are expressed as fold change \pm standard error of the mean compared with OC. The Western blot bands were quantified with densitometry using Image-J software (National Institutes of Health, Bethesda, Md). $P$ values for the Western blot data are reported from a 2-way analysis of variance followed by a post hoc Bonferroni test of the densitometry arbitrary units.

\section{RESULTS}

\section{Animal Model}

Animals included for analysis survived to the completion of the study. When compared with animals fed a regular diet (OC), animals fed a high-fat diet (OHC and OHCS) had a significant increase in weight from the time of ameroid placement to the time of harvest and significantly elevated glucose levels 30 minutes after an intraoperative dextrose challenge $(P<.001, P=.011$, respectively) (Figure 2). As previously published, capillary density and arteriolar density were significantly increased in the OHCS group 


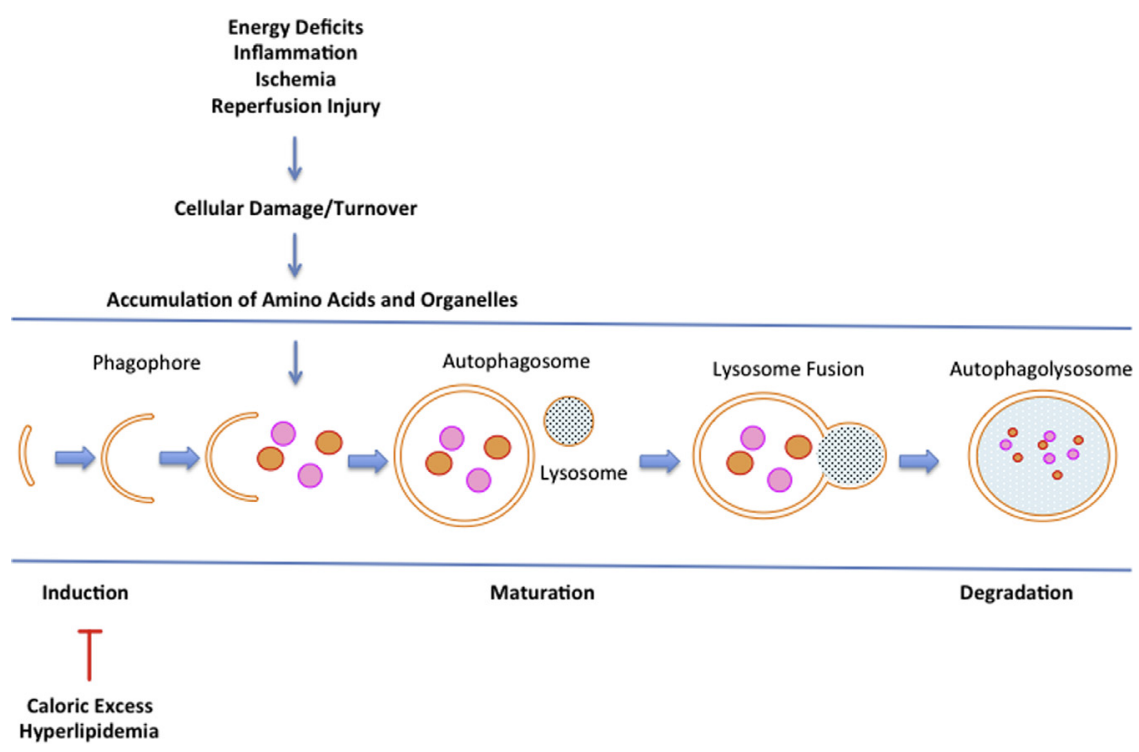

FIGURE 1. Schematic model of autophagy in the setting of cardiomyocyte stress.

compared with the OHC group. Despite this, there was no significant difference in myocardial perfusion or microvessel reactivity. ${ }^{21}$

\section{Western Blots}

Nonischemic myocardium. In NIM, animals in the OHC group had a relative increase in the autophagy flux marker P62 with a significant increase in the autophagy inhibitory protein mTOR $(P=.017)$ compared animals in the OC group. Animals in the OHC group also had an accumulation in several proteins essential for autophagy, including Beclin-1, LC3B-I, and LCB-II $(P=.025, P<.0001$, and $P=.0012$, respectively) (Table 1 , Figure 3 ). Of note, atorvastatin supplementation prevented these changes as demonstrated by a decrease in autophagy flux marker P62 (0.0056) and a decrease in mTOR $(P=.017)$ in the OHCS group compared with the OHC group. Animals in the OHCS group also had a relative decrease in Beclin-1 with a significant decrease in LC3B-I and LCB-II $(P<.001$ and $P=.0012$, respectively) (Table 1, Figure 3) compared with the OHC group.

Ischemic myocardium. In the IM, atorvastatin had the opposite effect, with a decrease in autophagy flux as indicated by an increase in p62 $(P=.0021)$ and an accumulation of LC3B-I (0.012), LCB-II, and LAMP-2 $(P=.012, P=.057$, and $P=.0095$, respectively $)$ (Table 1, Figure 3). Of note, neither a high-fat diet alone nor a high-fat diet with supplemental atorvastatin significantly affected mTOR signaling $(P=.095)$.

\section{DISCUSSION}

The results of this study demonstrate that in the setting of metabolic syndrome, atorvastatin supplementation has differential effects on autophagy, such that it is upregulated
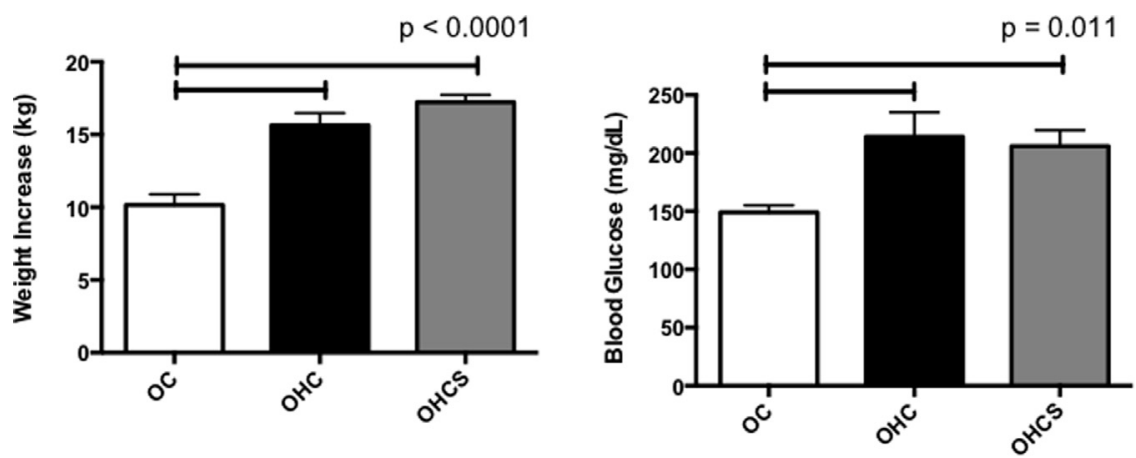

FIGURE 2. Weight increase (kilograms) and blood glucose after dextrose challenge at terminal procedure: Weight change and blood glucose level \pm standard error of the mean. Weights were measured before ameroid placement and again before harvest. Blood glucose level measured 30 minutes after intravenous dextrose challenge $(50 \mathrm{mg} / \mathrm{kg})$. One-way analysis of variance performed to determine $P$ value. Bonferroni multiple comparison tests were performed. $O C$, Regular diet control group; $O H C$, high cholesterol control group; $O H C S$, high cholesterol control group supplemented with daily oral atorvastatin. 
TABLE 1. Protein expression in myocardium

\begin{tabular}{lcccc}
\hline \multicolumn{1}{c}{ Targets } & OC & OHC & OHCS & P value \\
\hline NIM & & & & \\
P62 & $1 \pm 0.149$ & $1.30 \pm 0.211$ & $0.51 \pm 0.067$ & $.0056 \ddagger$ \\
mTOR & $1 \pm 0.110$ & $2.17 \pm 0.429$ & $1.09 \pm 0.240$ & $.017^{*}, \ddagger$ \\
Beclin-1 & $1 \pm 0.081$ & $1.74 \pm 0.256$ & $1.43 \pm 0.145$ & $.025^{*}$ \\
LC3B-I & $1 \pm 0.066$ & $3.04 \pm 0.365$ & $1.29 \pm 0.135$ & $<.001^{*} \ddagger$ \\
LC3B-II & $1 \pm 0.147$ & $1.86 \pm 0.240$ & $0.94 \pm 0.085$ & $.0012^{*} \ddagger$ \\
LAMP-2 & $1 \pm 0.093$ & $1.12 \pm 0.080$ & $0.83 \pm 0.115$ & .13 \\
IM & & & & \\
P62 & $1 \pm 0.053$ & $1.04 \pm 0.054$ & $1.54 \pm 0.158$ & $.0021 \dagger, \ddagger$ \\
mTOR & $1 \pm 0.200$ & $0.93 \pm 0.129$ & $0.98 \pm 0.130$ & .95 \\
Beclin-1 & $1 \pm 0.248$ & $1.07 \pm 0.229$ & $0.86 \pm 0.183$ & .79 \\
LC3B-I & $1 \pm 0.079$ & $0.58 \pm 0.064$ & $1.60 \pm 0.358$ & $.012 \ddagger$ \\
LC3B-II & $1 \pm 0.196$ & $0.45 \pm 0.063$ & $1.06 \pm 0.246$ & .057 \\
LAMP-2 & $1 \pm 0.257$ & $0.46 \pm 0.067$ & $1.92 \pm 0.389$ & $.0095 \ddagger$ \\
\hline
\end{tabular}

Protein expression listed as fold change \pm standard error of the mean compared with OC. One-way analysis of variance performed to determine $P$ value. Bonferroni multiple comparison tests were performed. IM, Ischemic myocardium; LAMP-2, lysosome-associated membrane protein 2; LC3B-I, light chain 3B-I; LC3B-II, light chain 3B-II; NIM, nonischemic myocardium; OC, regular diet control group; $O H C$, high cholesterol control group; OHCS, high cholesterol control group supplemented with daily oral atorvastatin; $m T O R$, mammalian target of rapamycin. *Indicates significant difference between the $\mathrm{OC}$ and $\mathrm{OHC}$ groups. †Indicates significant difference between the $\mathrm{OC}$ and OHCS groups. $\ddagger$ Indicates significant difference between the $\mathrm{OHC}$ and OHCS groups.

in NIM and downregulated in IM. The perioperative benefits of statins in patients undergoing cardiac surgery are significant, yet the underlying mechanisms remain under investigation. ${ }^{12,16}$ Autophagy has become increasingly implicated in cardioprotection during cardiac surgery, and several studies have demonstrated the significant effects of statins on autophagy. ${ }^{3,4}$ Significant potential therapies may be uncovered and optimized as we explore mechanisms by which statins regulate autophagy.

Autophagy is a complex and dynamic process with several points of regulation. It has become widely accepted that alterations in autophagy can be determined through the measurement of several regulatory protein markers. ${ }^{3,5,8}$ In particular, measuring alterations of P62 has been used as an index of autophagy flux. ${ }^{25}$ In response to different stressors or stimuli, P62 can be altered within minutes. ${ }^{3}$ We found that animals given a high-fat diet had an increase in regulatory markers of autophagy, including Beclin-1, LC3B, and LAMP2. These findings alone could suggest an increase in autophagy or a decrease in autophagy, because these proteins accumulate in the setting of decreased autophagy. However, measuring the autophagy flux marker P62 allows for a more clear understanding of the results. Given that P62 is degraded by the activation of autophagy, we can assume that the increase in P62 in this setting is a result of diminished autophagy. Further supporting this finding is the increase of mTOR. A known inhibitor of autophagy, mTOR has been demonstrated to be upregulated in the setting of caloric excess. ${ }^{8,9}$ In the
NIM, atorvastatin prevented these changes, resulting in an overall increase in autophagy.

In the IM, atorvastatin had a different effect, resulting in a relative decrease in autophagy as demonstrated by an increase in $\mathrm{P} 62$ and other regulatory proteins, including LC3B-I, LC3B-II, and LAMP-2. It remains unclear why atorvastatin had these differential effects in IM versus NIM. Further elucidating a reason for this difference may help provide insight into how statins improve outcomes in patients with coronary artery disease and how perioperative statin use can be optimized for cardioprotection. This difference suggests alternative mechanisms by which statins affect autophagy, depending on the stimulus and environment. Perhaps animals that were already receiving statin therapy had increased resistance to the stress of ischemia, resulting in a relative decreased need for autophagy to remove harmful by-products in the IM. Another possibility is that ischemic stress results in an overexpression of autophagy markers, and statin therapy helps maintain an appropriate homeostatic balance in the setting of increased oxidative stress and cell death. Indeed, autophagy in excess and impaired clearance of autophagosomes have been demonstrated to contribute to cardiomyocyte death. ${ }^{26,27}$

Mechanisms by which statins regulate autophagy remain under investigation. Several studies have demonstrated that the various forms of statins appear to have similar effects on autophagy through the inhibition of mTOR. ${ }^{18,28,29}$ Of note, inhibition of mTOR activity has been shown to impair angiogenesis, ${ }^{30}$ whereas prolonged mTOR activity can lead to vascular dysfunction. ${ }^{31}$ Sinha and colleagues ${ }^{32}$ demonstrated that inhibition of mTOR activity improves coronary microvascular flow after cardiac transplantation. During early stages of atherosclerosis, there is evidence that statins enhance autophagy through inhibition of mTOR, resulting in stabilization of coronary plaques through autophagymediated macrophage depletion and inhibition of cell death. In contrast, "mTOR independent autophagy" has been associated with increased inflammation and coronary plaque progression and instability. ${ }^{28,33}$ As already mentioned, in our current study, atorvastatin therapy regulated mTOR expression in the NIM, whereas in the IM there was no significant difference in mTOR regulation among the 3 groups. The stressful stimuli associated with chronic ischemia may have resulted in an overwhelming and relatively equivalent effect on mTOR signaling, regardless of diet modification. Again, this suggests a different mechanism for autophagy regulation in the IM that is mTOR independent. Alternate mechanisms by which statins may affect autophagy in an mTOR-independent manner are likely and merit further investigation.

\section{Study Limitations}

Foremost, we are unable to comment on differences in treatment length or dose-dependent responses because 


\section{Non-Ischemic Myocardium}
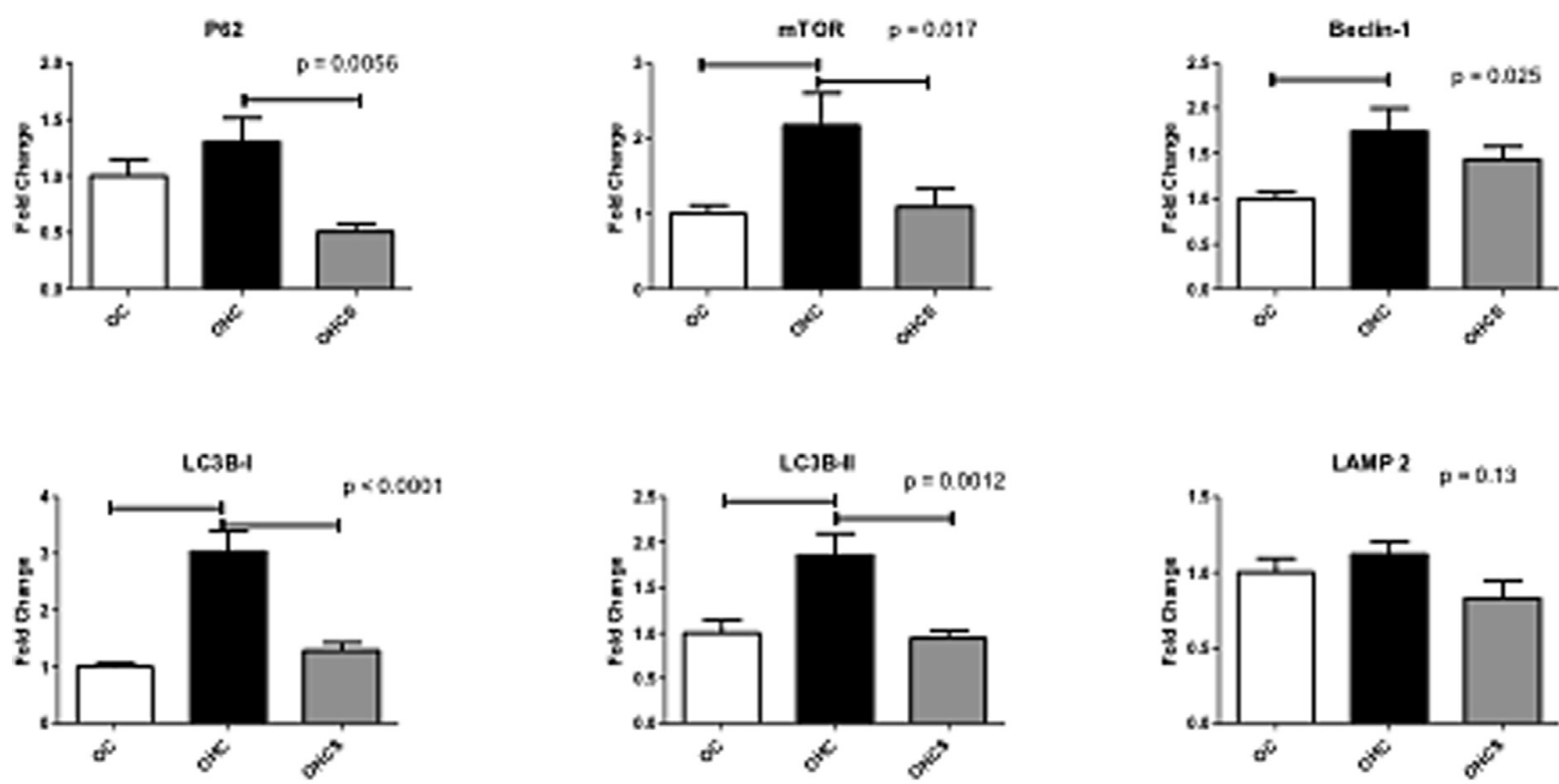

\section{Ischemic Myocardium}
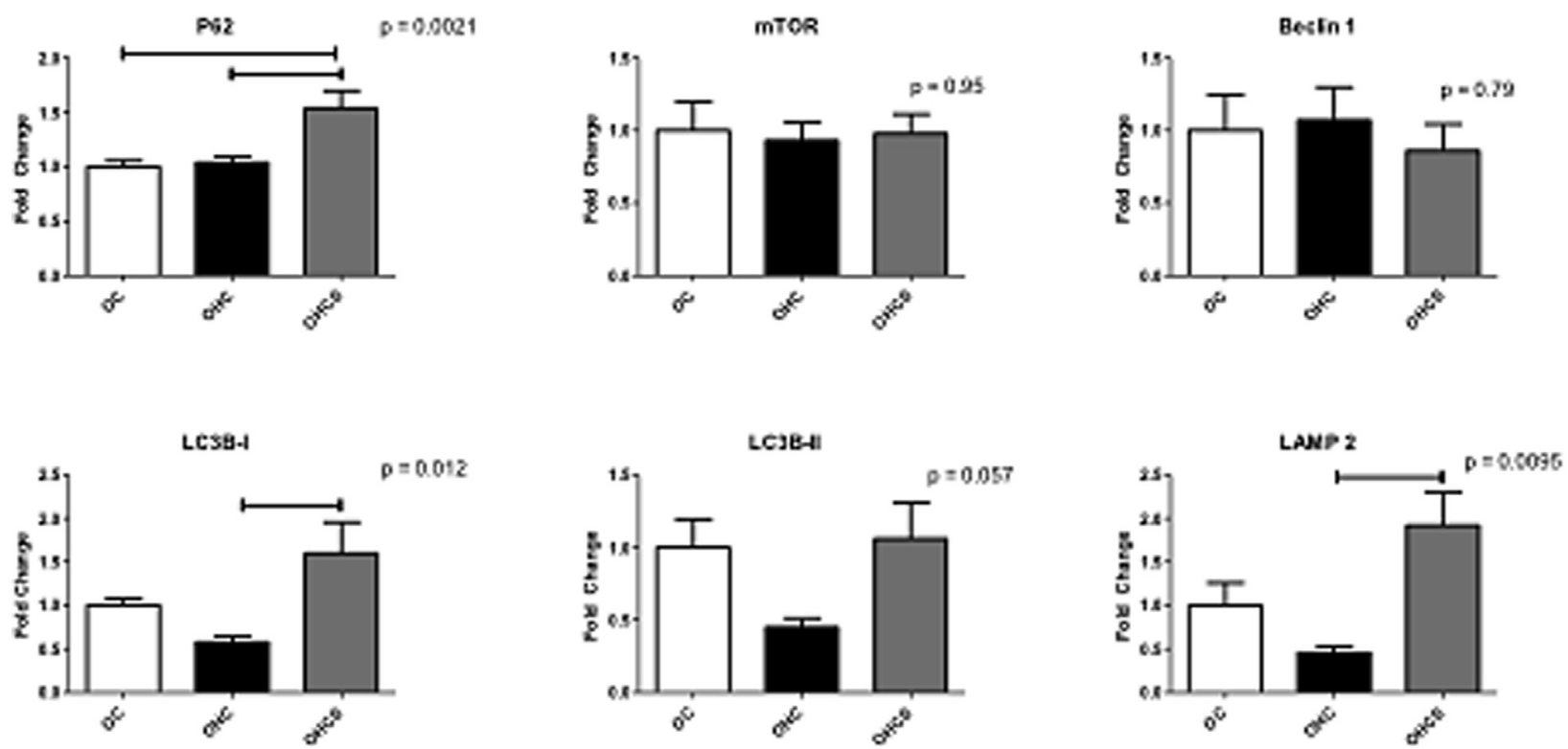

FIGURE 3. Protein expression in myocardium fold change \pm standard error of the mean compared with OC. One-way analysis of variance performed to determine $P$ value. Bonferroni multiple comparison tests were performed. $L A M P$-2, Lysosome-associated membrane protein 2; LC3B-I, light chain 3B-I; $L C 3 B-I I$, light chain 3B-II; mTOR, mammalian target of rapamycin; OC, regular diet control group; OHC, high cholesterol control group; OHCS, high cholesterol control group supplemented with daily oral atorvastatin.

treatment length and atorvastatin doses were fixed. Because autophagy is a dynamic process, it is important to note that levels of autophagy markers may differ from our current results if tissue was analyzed at different time-points. Indeed, this is a limitation when using a large animal model, in which the least number of animals are used to achieve adequate power. It is also important to note that we did not directly measure autophagy activity. The gold standard for directly measuring autophagy is by directly visualizing the autolyosome at different stages of its development. Because autophagy is a ubiquitous process and the autolysosome easily can be mistaken for other structures, electron microscopy would not allow any reasonable means to objectively and reproducibly quantify the process. These 
are known criticisms for using electron microscopy. Thus, as has been described by our group and others, ${ }^{3,5,8}$ we used Western blotting to measure specific and essential markers of the process. Although this method is an indirect measure of autophagy, it allows for the objective measurement and reproducibility necessary to conduct a study of this nature.

Further complicating the study of statins is the known dose-dependent effects of this statins. Urbich and colleagues $^{34}$ demonstrated that atorvastatin has differential dose-dependent effects on endothelial cell migration and angiogenesis, where lower doses resulted in improved indicators of angiogenesis and higher doses resulted in prevention of migration through endothelial cell apoptosis. We have also noted a dose-dependent effect of atorvastatin on indicators of angiogenesis, where a lower dose of atorvastatin resulted in an upregulation of proangiogenic proteins along with increased capillary and arteriolar densities, and higher doses of atorvastatin did not improve angiogenic response. ${ }^{21,35}$ In the current study, we again used a relatively lower dose than in our prior studies, giving $1.5 \mathrm{mg} / \mathrm{kg}$ of atorvastatin. However, this is still a relatively high dose of atorvastatin, and an even lower dose may have exposed different effects.

\section{CONCLUSIONS}

This represents the first study in a large animal model to demonstrate the effects of atorvastatin on autophagy in chronic myocardial ischemia. Of note, and distinct from many other animal studies, this study was performed in animals provided with several months of a high-fat diet resulting in significantly increased weight gain and signs of insulin resistance (Figure 2). Given the continued increase in obesity and diabetes, and the widespread use of statins, this study highlights important effects of statins in a clinically relevant large animal model. The findings of this study may have implications regarding the purported protective role of statin drugs in the setting of cardiac surgery. Further investigation into the effects of statins on autophagy in IM and NIM is warranted.

\section{References}

1. Gustafsson AB, Gottlieb RA. Recycle or die: the role of autophagy in cardioprotection. J Mol Cell Cardiol. 2008;44:654-61.

2. Gottlieb RA, Finley KD, Mentzer RM Jr. Cardioprotection requires taking out the trash. Basic Res Cardiol. 2009;104:169-80.

3. Jahania SM, Sengstock D, Vaitkevicius P, Andres A, Ito BR, Gottlieb RA, et al. Activation of the homeostatic intracellular repair response during cardiac surgery. J Am Coll Surg. 2013;216:719-29.

4. Garcia L, Verdejo HE, Kuzmicic J, Zalaquett R, Gonzalez S, Lavandero S, et al. Impaired cardiac autophagy in patients developing postoperative atrial fibrillation. J Thorac Cardiovasc Surg. 2012;143:451-9.

5. Klionsky DJ, Abdalla FC, Abeliovich H, Abraham RT, Acevedo-Arozena A, Adeli K, et al. Guidelines for the use and interpretation of assays for monitoring autophagy. Autophagy. 2012;8:445-544.

6. Yan WJ, Dong HL, Xiong LZ. The protective roles of autophagy in ischemic preconditioning. Acta Pharmacol Sin. 2013;34:636-43.
7. Yan L, Vatner DE, Kim SJ, Ge H, Masurekar M, Massover WH, et al. Autophagy in chronically ischemic myocardium. Proc Natl Acad Sci U S A. 2005;102: 13807-12.

8. Sabe AA, Elmadhun NY, Dalal RS, Robich MP, Sellke FW. Resveratrol regulates autophagy signaling in chronically ischemic myocardium. J Thorac Cardiovasc Surg. 2014;147:792-9.

9. Glazer HP, Osipov RM, Clements RT, Sellke FW, Bianchi C. Hypercholesterolemia is associated with hyperactive cardiac mTORC1 and mTORC2 signaling. Cell Cycle. 2009;8:1738-46.

10. Schwartz Longacre L, Kloner RA, Arai AE, Baines CP, Bolli R, Braunwald E et al. New horizons in cardioprotection: recommendations from the 2010 National Heart, Lung, and Blood Institute Workshop. Circulation. 2011;124 1172-9.

11. Kulik A, Brookhart MA, Levin R, Ruel M, Solomon DH, Choudhry NK. Impact of statin use on outcomes after coronary artery bypass graft surgery. Circulation. 2008; 118:1785-92.

12. Howard PA, Barnes BJ. Potential use of statins to prevent atrial fibrillation after coronary artery bypass surgery. Ann Pharmacother. 2008;42:253-8.

13. Clark LL, Ikonomidis JS, Crawford FA Jr, Crumbley A III, Kratz JM, Stroud MR, et al. Preoperative statin treatment is associated with reduced postoperative mortality and morbidity in patients undergoing cardiac surgery: an 8-year retrospective cohort study. J Thorac Cardiovasc Surg. 2006;131: $679-85$.

14. Lazar HL, Bao Y, Zhang Y, Bernard SA. Pretreatment with statins enhances myocardial protection during coronary revascularization. J Thorac Cardiovasc Surg. 2003; 125:1037-42.

15. Pan W, Pintar T, Anton J, Lee VV, Vaughn WK, Collard CD. Statins are associated with a reduced incidence of perioperative mortality after coronary artery bypass graft surgery. Circulation. 2004;110:II45-9.

16. Ouattara A, Benhaoua H, Le Manach Y, Mabrouk-Zerguini N, Itani O Osman A, et al. Perioperative statin therapy is associated with a significant and dose-dependent reduction of adverse cardiovascular outcomes after coronary artery bypass graft surgery. J Cardiothorac Vasc Anesth. 2009;23: 633-8.

17. Andres AM, Hernandez G, Lee P, Huang C, Ratliff EP, Sin J, et al. Mitophagy is required for acute cardioprotection by simvastatin. Antioxid Redox Signal. September 20, 2013 [Epub ahead of print].

18. Martinet W, De Loof H, De Meyer GR. mTOR inhibition: a promising strategy for stabilization of atherosclerotic plaques. Atherosclerosis. 2014;233:601-7.

19. Kocsis GF, Pipis J, Fekete V, Kovacs-Simon A, Odendaal L, Molnar E, et al Lovastatin interferes with the infarct size-limiting effect of ischemic preconditioning and postconditioning in rat hearts. Am J Physiol Heart Circ Physiol. 2008;294:H2406-9.

20. Weis M, Heeschen C, Glassford AJ, Cooke JP. Statins have biphasic effects on angiogenesis. Circulation. 2002;105:739-45.

21. Elmadhun NY, Lassaletta AD, Chu LM, Liu Y, Feng J, Sellke FW Atorvastatin increases oxidative stress and modulates angiogenesis in Ossabaw swine with the metabolic syndrome. J Thorac Cardiovasc Surg. 2012;144: 1486-93.

22. Lassaletta AD, Chu LM, Robich MP, Elmadhun NY, Feng J, Burgess TA, et al. Overfed Ossabaw swine with early stage metabolic syndrome have normal coronary collateral development in response to chronic ischemia. Basic Res Cardiol. 2012;107:243.

23. Elmadhun NY, Sabe AA, Robich MP, Chu LM, Lassaletta AD, Sellke FW The pig as a valuable model for testing the effect of resveratrol to prevent cardiovascular disease. Ann N Y Acad Sci. 2013;1290:130-5.

24. Vilahur G, Padro T, Badimon L. Atherosclerosis and thrombosis: insights from large animal models. J Biomed Biotechnol. 2011;2011:907575.

25. Bjorkoy G, Lamark T, Pankiv S, Overvatn A, Brech A, Johansen T. Monitoring autophagic degradation of p62/SQSTM1. Methods Enzymol. 2009;452:181-97.

26. Gustafsson AB, Gottlieb RA. Autophagy in ischemic heart disease. Circ Res. 2009;104:150-8

27. Ma X, Liu H, Foyil SR, Godar RJ, Weinheimer CJ, Hill JA, et al. Impaired autophagosome clearance contributes to cardiomyocyte death in ischemia/ reperfusion injury. Circulation. 2012;125:3170-81.

28. Wei YM, Li X, Xu M, Abais JM, Chen Y, Riebling CR, et al. Enhancement of autophagy by simvastatin through inhibition of Rac1-mTOR signaling pathway in coronary arterial myocytes. Cell Physiol Biochem. 2013;31:925-37.

29. Wagner RJ, Martin KA, Powell RJ, Rzucidlo EM. Lovastatin induces VSMC differentiation through inhibition of Rheb and mTOR. Am J Physiol Cell Physiol. 2010;299:C119-27. 
30. Humar R, Kiefer FN, Berns H, Resink TJ, Battegay EJ. Hypoxia enhances vascular cell proliferation and angiogenesis in vitro via rapamycin (mTOR)-dependent signaling. FASEB J. 2002;16:771-80.

31. Mancini D, Pinney S, Burkhoff D, LaManca J, Itescu S, Burke E, et al. Use of rapamycin slows progression of cardiac transplantation vasculopathy. Circulation. 2003:108:48-53.

32. Sinha SS, Pham MX, Vagelos RH, Perlroth MG, Hunt SA, Lee DP, et al. Effect of rapamycin therapy on coronary artery physiology early after cardiac transplantation. Am Heart J. 2008;155:889.e1-6.
33. Lavandero S, Troncoso R, Rothermel BA, Martinet W, Sadoshima J, Hill JA. Cardiovascular autophagy: concepts, controversies, and perspectives. Autophagy. 2013;9:1455-66.

34. Urbich C, Dernbach E, Zeiher AM, Dimmeler S. Double-edged role of statins in angiogenesis signaling. Circ Res. 2002;90:737-44.

35. Boodhwani M, Mieno S, Voisine P, Feng J, Sodha N, Li J, et al. High-dose atorvastatin is associated with impaired myocardial angiogenesis in response to vascular endothelial growth factor in hypercholesterolemic swine. J Thorac Cardiovasc Surg. 2006;132:1299-306.

\title{
EDITORIAL COMMENTARY
}

\section{Cardiac cannibalism}

\author{
Alden H. Harken, MD, FACS
}

See related article on pages 3172-8.

So, if you and a couple of guys are floating on a life raft in the middle of the Pacific and you are running out of food, what do you do? You throw the guy who eats the most overboard. Unless, of course, you can swallow both your pride and the big guy's liver. As it turns out, Drosophila figured out both of these steps millions of years ago ... and autophagy has been evolutionarily conserved ever since. This remarkable self-preservation strategy is activated by both starvation and stress.

Autophagy is a catabolic mechanism of degrading and disposing of dysfunctional cellular elements. Broken or profligate cytoplasmic constituents are herded into and isolated in a double-membraned vesicle imaginatively termed an autophagosome. This vesicle then fuses with a toxic, enzyme-laden lysosome, which spews its contents into the autophagosomal recycling container. The component parts of the previously worn-out cytoplasmic scaffolding can then be used to rebuild essential cellular machinery. But-like many good things-the autophagosome can go rogue and begin eating things that are not on the menu.

From the Department of Surgery, University of California, San Francisco-East Bay, Oakland, Calif.

Disclosures: Author has nothing to disclose with regard to commercial support.

Received for publication Sept 19, 2014; accepted for publication Sept 20, 2014; available ahead of print Oct 16, 2014.

Address for reprints: Alden H. Harken, MD, FACS, Department of Surgery, University of California, San Francisco-East Bay, 1411 E 31st St, QIC 22134, Oakland, CA 94602 (E-mail: alden.harken@ucsfmedctr.org).

J Thorac Cardiovasc Surg 2014;148:3178-9

$0022-5223 / \$ 36.00$

Copyright (c) 2014 by The American Association for Thoracic Surgery

http://dx.doi.org/10.1016/j.jtcvs.2014.09.070
In the study described in their article in this issue of the Journal of Thoracic and Cardiovascular Surgery, Sabe and colleagues ${ }^{1}$ from the Sellke laboratory fed Ossabaw pigs a regular diet, a high-cholesterol diet, or the same high-cholesterol pâté plus a statin. The lipid-laden diet contained a "high-cholesterol" formula consisting of $4 \%$ cholesterol, $17.2 \%$ coconut oil, $2.3 \%$ corn oil, $1.5 \%$ sodium cholate, and $75 \%$ regular chow. ${ }^{2}$ Let's place this in perspective: a McDonald's Double Quarter Pounder With Cheese provides 277 grams of enjoyment with 749 calories, 42 grams of fat, and $165 \mathrm{mg}$ of cholesterol. ${ }^{3}$ So the pudgy pigs in this study got the equivalent of a couple of Quarter Pounders a day and probably thought they were starving. And these pigs paid the price of cholesterolboosting gluttony with an associated decrease in protective autophagy.

Interestingly, atorvastatin (not available to most pigs) preserved this highly-conserved evolutionary myocellular protective mechanism in nonischemic myocardium. Perhaps surprisingly, a statin had the opposite effect in the ischemic heart.

Several aspects of this important article are fascinating: (1) for those of us type A cardiac surgical ascetics who are capable of sticking to a lifetime relatively healthy diet, in only 14 weeks of binge eating, we can blow the whole thing; (2) statins, which block the rate-limiting step in cholesterol synthesis, are also termed pleiotropic (which means that they also do lots of stuff that we don't understand); (3) for healthy nonischemic myocardium, autophagy seems to be an effective depository for component recycling and energy; and (4) a cardiomyocellular autophagosome in a male Ossabaw pig is kind of like the garbage disposal in the kitchen of a recycling center run by solar panels on the roof. 